\title{
LA TECNOLOGIA DEI SOCIAL NETWORK E LA GESTIONE DELLE PERSONE NEL RECLUTAMENTO E SELEZIONE DEI PROPRI DIPENDENTI
}

\section{ARTICOLO ORIGINALE}

SUMAR, Ramiro Rodrigues ${ }^{1}$

SUMAR, Ramiro Rodrigues. La tecnologia dei social network e la gestione delle persone nel reclutamento e selezione dei propri dipendenti. Revista Científica Multidisciplinar Núcleo do Conhecimento. Anno 06, Ed. 07, Vol. 12, pp. 74-90. Luglio 2021. ISSN: 2448-0959, Link di accesso: https://www.nucleodoconhecimento.com.br/economia-aziendale/dipendenti, $\quad$ DOI: 10.32749/nucleodoconhecimento.com.br/economia-aziendale/dipendenti

\section{RIEPILOGO}

Obiettivo: Descrivere l'impatto che i social network possono avere sul reclutamento e sulla selezione dei propri dipendenti. Problema della domanda: In che modo il social network può favorire il reclutamento e la selezione dei dipendenti di un'azienda? Metodologia: revisione della letteratura. Risultati: L'evidenza dei risultati ha dimostrato che le tecnologie attraverso i social network possono essere rilevanti per il reclutamento e la selezione di persone per l'organizzazione. Ma questo reclutamento dovrebbe essere fatto con uno sguardo differenziato su ogni tipo di social network da parte del reclutatore. Considerazioni finali: il reclutamento e la selezione sono stati cambiando come un modo tradizionale (faccia a faccia) per la modalità tecnologica (virtuale). Lo studio ha detto che i social network sono strumenti in grado di portare al reclutatore candidati in grado di prendere l'organizzazione in modo responsabile e che non ci sono barriere nel mondo virtuale per trovare il

\footnotetext{
${ }^{1}$ Laurea magistrale in Controllo, Specialista in Gestione Tecnologica, Amministrazione Strategica, Sicurezza delle Informazioni e Laureato in Scienze Contabili.
}

RC: 93823

Disponível em: https://www.nucleodoconhecimento.com.br/economia- 
candidato ideale. Si sottolinea l'importanza di estendere questo studio sulla base di prove scientifiche, in cui la ricerca può essere condotta in aziende per l'uso dei social network nel monitoraggio dei propri dipendenti.

Parole chiave: Gestione delle persone, Social network, Tecnologie dell'informazione e della comunicazione.

\section{INTRODUZIONE}

Le aziende hanno nei loro obiettivi principali di ottenere risultati, tuttavia, perché ciò accada, il primo passo è assumere professionisti qualificati e competenti per far parte del team. Tra i reparti che compongono un'azienda c'è la parte della gestione delle risorse umane, che ha come una delle sue funzioni quella di assumere per l'organizzazione il proprio personale professionale (CARVALHO, 2015).

La parola "amministrare" consiste nel processo decisionale e nella realizzazione di azioni (collaborative, per migliorare le risorse, l'interazione, tra gli altri) in modo da poter raggiungere gli obiettivi desiderati dall'azienda (COSTA, 2018). Pertanto, il focus della gestione delle risorse umane (o della gestione delle persone) è legato al rendimento delle competenze, tra cui: efficienza, efficacia, produttività, soddisfazione e impegno verso le persone, sopravvivenza dell'organizzazione, qualità, competitività e responsabilità sociale (SPADER, 2019).

II manager ha bisogno di avere diverse qualità per poter elencare la sua azienda, tra cui la leadership, la responsabilità e l'empatia per mediare le prestazioni dei suoi dipendenti, per questo ha bisogno di sapere come ascoltare ed essere aperto ai suggerimenti, essere partecipativo e partecipare, principalmente, ai compiti cercando di essere complice della delega di questi (DE BEM NORO; STUKER; DE OLIVEIRA, 2015).

RC: 93823

Disponível em: https://www.nucleodoconhecimento.com.br/economia- 
Secondo Ribeiro (2017), il responsabile delle risorse umane o, responsabile delle persone, oltre ad essere qualificato per la posizione, deve avere strumenti (valutazione delle prestazioni; feedback continuo; tecnologie dell'informazione e della comunicazione, tra le altre), necessarie per il raggiungimento di una buona amministrazione, considerando che la parte delle risorse umane è dove verranno eseguiti vari processi in modo che l'azienda possa garantire il proprio nome nel mercato aziendale, processi che mirano ad assumere dipendenti per completare il sistema organizzativo.

Questo reclutamento avviene in diverse forme, la più utilizzata oggi è attraverso le tecnologie. L'era tecnologica per le organizzazioni ha l'obiettivo di migliorare la qualità del servizio portando velocità nello sviluppo di prodotti/ servizi, affidabilità nei dati aziendali, maggiori prestazioni da parte dei dipendenti tra le altre qualità $e$, garantendo maggiore sicurezza ai propri utenti. Nella gestione delle persone, le tecnologie favoriscono una maggiore portata delle persone da reclutare, oltre ad aiutare anche il manager nel momento di conoscere davvero la vita personale dei suoi dipendenti (SILVA, 2019; PORTO et al., 2016; SANTOS, 2018).

Secondo Motta et al. (2017), la rete mondiale di computer (internet) favorisce che le relazioni tra dipendenti e manager possano costruire nuove culture di relazione e conoscenza, che possono derivare dalle conoscenze e dalle informazioni derivate dall'accesso a Internet, con maggiore intensità di social network accessibili o meno nell'ambiente di lavoro.

È essenziale comprendere che la gestione delle persone ha svolto un ruolo importante all'interno delle organizzazioni. Questo ruolo è modellato non solo su politiche e pratiche, ma anche cercando di evidenziare in fattori organizzativi problemi connessi come: tensioni tra lavoratori, cattiva organizzazione all'interno dell'ambiente di lavoro, problemi culturali che caratterizzano i pregiudizi, al fine di poter controllare i danni causati da una possibile molestia morale e che

RC: 93823

Disponível em: https://www.nucleodoconhecimento.com.br/economia- 
influenzeranno l'intera organizzazione dell'azienda (LEAL; MATOS; FONTANA, 2013).

Lo studio in questione mette in primo piano l'importanza delle tecnologie per l'assunzione e la selezione dei dipendenti che cercano di lavorare in un'organizzazione. La giustificazione di questo studio arriva dal ricercatore che ha esperienza nelle tecnologie dell'informazione e della comunicazione e osserva che nei social network l'offerta di lavoro nasce con una domanda molto significativa, così come l'osservazione del modo in cui si terrà il colloquio (in particolare le persone di livello superiore il colloquio avviene da google meet). In questo segmento, la domanda problematica di questo studio è: Come può il social network favorire il reclutamento e la selezione dei dipendenti di un'azienda?

Pertanto, il contributo principale alla realizzazione di questo studio si basa sulla conoscenza dell'uso delle tecnologie da parte dei people manager, dai social network, rendendo rilevante aggiungere maggiori conoscenze sia al professionista che alla società in generale. Per la comunità scientifica, lo studio ha la sua rilevanza aggiunta all'espansione delle conoscenze sull'uso delle tecnologie per il reclutamento e la selezione delle persone.

L'obiettivo proposto in questo studio era quello di descrivere l'impatto che i social network possono avere sul reclutamento e sulla selezione dei loro dipendenti.

Affinché questo studio si svolge, abbiamo cercato una base teorica relativa ai seguenti temi: Reclutamento e selezione in azienda; tecnologie dell'informazione che hanno un impatto sulla gestione delle persone; Social network per il reclutamento e la selezione di un'azienda.

Sulla base di questa base teorica, la progettazione dello studio ha la sua metodologia basata sulla rassegna bibliografica.

RC: 93823

Disponível em: https://www.nucleodoconhecimento.com.br/economia- 


\section{RIFERIMENTO TEORICO}

\subsection{IL RUOLO DELL'ASSUNZIONE E DELLA SELEZIONE NELL'AZIENDA}

Parlando di reclutamento e selezione delle persone, Oliveira e Bueno (2018) descrivono che questo sistema di scelta viene dagli uomini delle caverne, dove le scelte delle persone per sviluppare le attribuzioni imposte dal sistema sociale dell'epoca sono state date dalla loro forza, essendo la condizione più forte per partecipare alle cacce per l'alimentazione del loro popolo. Nel contesto attuale, questa selezione sta diventando sempre più difficile.

Chiavenato (2002) menziona l'importanza del reclutamento e della selezione per l'organizzazione. L'autore rafforza che entrambi gli strumenti di scelta organizzativa sono direttamente collegati allo sviluppo dell'azienda e, anche con attribuzioni diverse, hanno lo stesso obiettivo: identificare il dipendente appropriato per compongono l'organizzazione.

L'assunzione può essere classificata come interna o esterna. II reclutamento interno avviene quando l'organizzazione apporta una modifica al proprio personale. Questo cambiamento deriva dalle prestazioni del lavoratore nel suo settore e dalle esigenze dell'azienda, che consente la scelta di questo lavoratore che è stato osservato per qualche tempo, dalla promozione, cioè dal trasferimento in reparti o posizioni con maggiore risalto. Da questa modifica questo dipendente viene formato, in modo che le sue prestazioni possano ottenere risultati migliori all'interno dell'azienda con le sue attività (CANALLI; DE ANDRADE, 2016).

Nel reclutamento esterno, il dipendente può essere scelto attraverso processi o con altri mezzi. Esempi in relazione a questi media sono gli annunci sulla stampa, le lezioni nelle scuole e nelle università, la diffusione in associazioni di classe, le raccomandazioni di terzi, le agenzie di collocamento, i file dei candidati memorizzati

RC: 93823

Disponível em: https://www.nucleodoconhecimento.com.br/economia- 
nell'azienda che si sono già presentati e lo scambio di aziende. Nel reclutamento esterno, gli annunci dell'azienda e le pagine di diffusione dei posti vacanti su Internet portano questa notorietà che i dipendenti sono necessari (SILVA; FERRETT; MANCINI, 2014).

Pertanto, tutti gli sforzi dell'azienda per portare alla sua cerchia nuovi dipendenti sono legati al reclutamento. Alberton, (2002), descrive che il processo di assunzione è considerato una delle fasi più importanti del processo di ammissione di un nuovo professionista. L'autore rafforza che la maggior parte delle aziende cerca in primo piano all'interno dell'ambiente interno, per poi andare al mercato del lavoro.

Mozer (2016) rafforza che l'organizzazione ha culture diverse l'una dall'altra. Queste culture possono far sì che il lavoro venga svolto in modi diversi. Tuttavia, l'assunzione e la selezione non sono diverse. II processo attraverso il quale viene effettuata l'assunzione dell'organizzazione ha le sue variazioni, ma i mezzi utilizzati sono gli stessi, e possono essere attraverso le tecnologie dell'informazione e della comunicazione, dove i posti vacanti sono divulgati per posizioni all'interno dell'azienda. Nel caso delle selezioni, c'è una decisione con cui il candidato si posizionerà dalla migliore prestazione alla posizione che gli viene offerta.

Sia l'assunzione in forma interna che esterna presenta vantaggi e svantaggi. Per l'organizzazione e per i concorrenti della posizione resa disponibile nell'impresa, queste condizioni dovrebbero essere esaminate per individuare il modo migliore per assumere. Le figure 1 e 2 fanno un'affermazione di questi vantaggi e svantaggi delle assunzioni interne ed esterne.

RC: 93823

Disponível em: https://www.nucleodoconhecimento.com.br/economia- 
Figura 1: Vantaggi delle assunzioni interne ed esterne

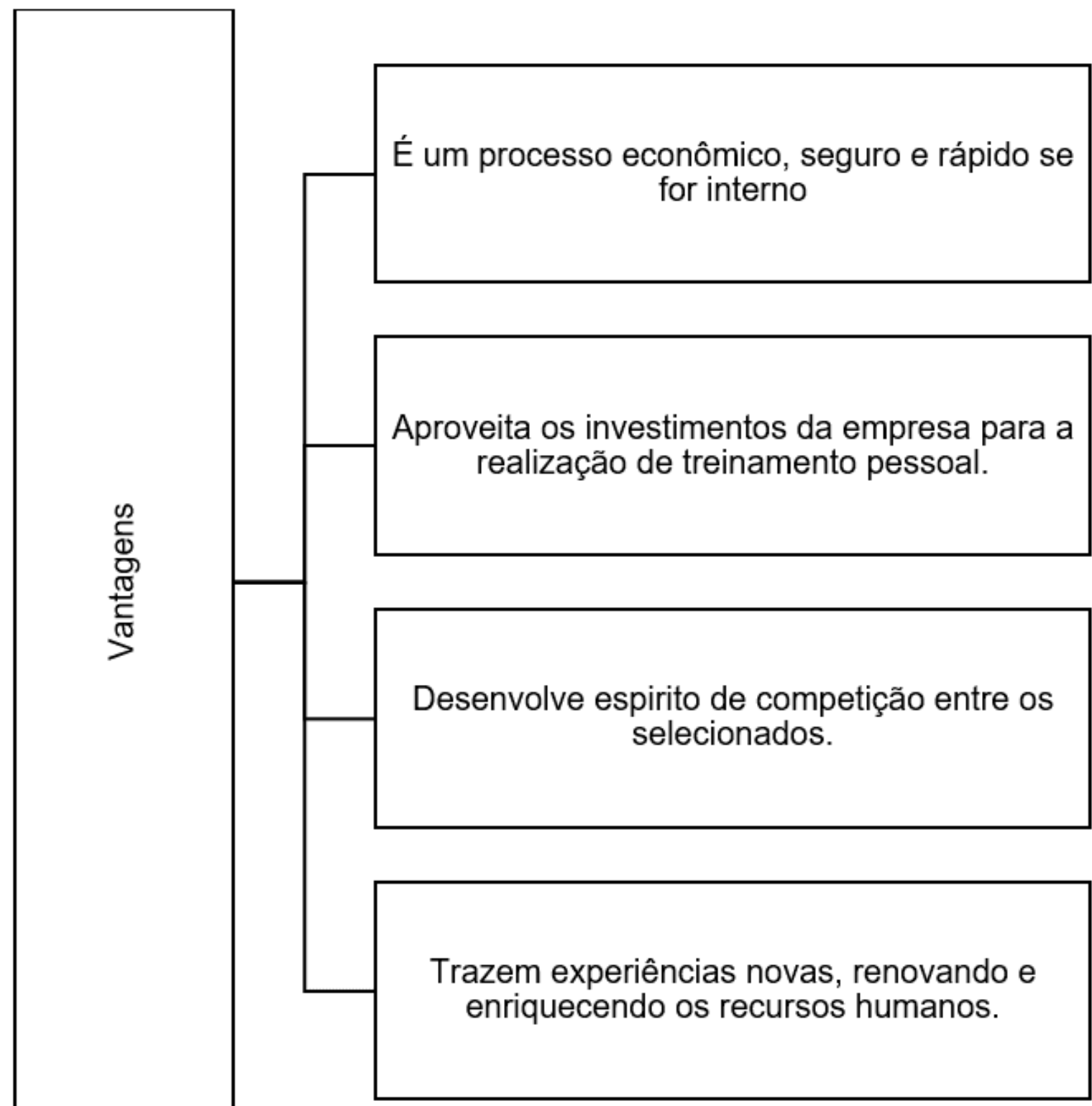

Fonte: Tratto da Chiavenato (2002).

RC: 93823

Disponível em: https://www.nucleodoconhecimento.com.br/economia- 
Figura 2: Svantaggi delle assunzioni interne ed esterne



Fonte: Tratto da Chiavenato (2002).

RC: 93823

Disponível em: https://www.nucleodoconhecimento.com.br/economia- 
Pertanto, il reclutamento e la selezione sono strumenti in cui le aziende sono disposte a investire, aggiungendo valore agli appaltatori. Pertanto, i dipendenti selezionati devono essere in grado di svolgere la funzione per la quale il posto vacante ha gareggiato e conoscere le politiche che l'azienda adotta.

Chiavenato (2002) afferma che questi strumenti possono essere utilizzati in parallelo. La scelta del modo in cui dovrebbe essere utilizzato dipenderà dalla situazione, dal numero di candidati, dal profilo desiderato e, soprattutto, dai requisiti necessari per lo svolgimento delle funzioni.

È degno di nota che non tutti gli ambienti forniscano la condizione di formare leader, questa azione sviluppata dalla direzione delle persone rende possibile il reclutamento interno attraverso la leadership, che il dipendente ha autonomia e processo decisionale davanti all'altro team, armonizzando così l'ambiente, lasciandolo adeguato e principalmente in grado di aumentare la redditività e la produttività degli altri (LEITE, 2019).

\subsection{OTTIMIZZAZIONE DELLA GESTIONE DELLE PERSONE MEDIANTE LA GOVERNANCE IT}

La gestione delle persone nel corso degli anni ha subito adattamenti derivanti da profonde trasformazioni che possono comportare l'implementazione di azioni per ottenere risultati. Secondo Wanzerller et al. (2018), l'origine della gestione del nome deriva dal latino "gestione", che ne definisce il significato come atto o effetto di gestione o gestione o gestione e persona del latino "persona" che indica un uomo o una donna.

Dagli anni '80, la globalizzazione ha assunto lo scenario economico, con l'evoluzione delle comunicazioni, lo sviluppo tecnologico e la competitività. Questo avvento tecnologico ha portato diverse aziende ad acquisire nuove conoscenze sulle tecnologie dell'informazione nelle organizzazioni. Pertanto, la gestione delle persone

RC: 93823

Disponível em: https://www.nucleodoconhecimento.com.br/economia- 
è una funzione manageriale che copre la partecipazione delle persone allinterno delle organizzazioni al fine di raggiungere gli obiettivi e gli obiettivi organizzativi e individuali desiderati (POCHMANN, 2015; STECCA, 2014).

Pessoa et al. (2017, p. 02), descrive che:

Oggi è importante che le organizzazioni utilizzino strumenti tecnologici per risolvere il supporto alla propria attività. Con il progresso tecnologico, gli strumenti sono diventati potenti e in una certa misura accattivanti, facilità d'uso e integrazione con vari sistemi. I professionisti devono utilizzare strumenti per assisterli nella vita quotidiana dell'azienda.

Affinché l'azienda abbia un buon sviluppo è necessario che tutti i reparti siano allineati, con un manager attivo in ogni reparto. II reparto gestione del personale esercita grande importanza per un'organizzazione, perché attraverso di essa viene reclutato e selezionato l'intero team di lavoro, oltre a coinvolgere tutto ciò che è collegato all'azienda, sia internamente che esternamente: ambiente, politiche, società e concorrenti (REIS et al., 2018).

In tutto il mondo, le aziende guardano attraverso strumenti tecnologici, un modo per massimizzare il proprio business, concentrandosi sulle loro prestazioni, efficacia, soddisfazione del cliente, redditività ed efficienza del servizio. All'interno dei sistemi aziendali possiamo percepire che le forme valutative stanno consolidando il lavoro svolto dalla people management, un concetto ampiamente difeso da diversi autori come parla Lima e Figueiredo (2020) nell'elaborare la tesi secondo cui solo valutando ciò che è stato fatto nel corso di un dato tempo può essere identificata la produttività raggiunta nei settori.

In questo senso, il miglioramento della qualità delle organizzazioni negli ultimi decenni attraverso le tecnologie, ha portato benefici al mercato aziendale, come: ottimizzazione dei servizi, migliori prestazioni dei dipendenti, competitività, massimizzazione dei risultati tra gli altri (OLIVEIRA; VASCONCELOS, 2005). In questo contesto, il governo societario è diventato la struttura che mira a garantire le

RC: 93823

Disponível em: https://www.nucleodoconhecimento.com.br/economia- 
decisioni in relazione all'organizzazione, garantendo così gli interessi degli stakeholder (BARROS; DA SILVA, GOESE, 2015).

La governance IT ha portato innovazione alla gestione delle persone. Novato (2014) afferma che la governance IT è stata classificata come un insieme di elementi che portano pratiche, standard e relazioni strutturate, che sono diretti da manager e tecnici IT, con l'obiettivo di garantire controlli organizzativi, espandere i processi di prestazioni e sicurezza, ridurre al minimo i rischi e ottimizzare l'applicazione delle risorse, che porta l'IT in un allineamento guidato dal business.

Pertanto, le nuove tecnologie suggeriscono trasformazioni non solo nei processi aziendali, ma anche nel modo in cui vengono creati e commercializzati prodotti e servizi, nella struttura e negli obiettivi dell'azienda, nelle dinamiche della concorrenza e nella natura stessa del business e dei suoi dipendenti (TIDD; BESSANTE, 2015; KON, 2016).

Pertanto, la governance IT ha indicato come uno strumento efficace e sicuro nella gestione delle persone per la formattazione della struttura organizzativa, coinvolgendo la definizione delle persone per raggiungere il quadro professionale dell'organizzazione, in modo che questa divisione armonica del lavoro possa essere sviluppata da questo team con responsabilità che mira in comune alla crescita dell'azienda (LORENZETTI et al., 2014).

\subsection{SOCIAL NETWORK PER L'ASSUNZIONE E LA SELEZIONE DI UN'AZIENDA}

Quando si riferiscono le tecnologie dell'informazione e della comunicazione al reclutamento di persone, si dice che le tecnologie abbiano facilitato la vita di molte organizzazioni, assistendo nella selezione di buoni professionisti. II potenziale delle tecnologie, per una buona assunzione e selezione, viene sempre più utilizzato. Dos Santos; De Oliveira e Centurión (2018) indicanoi come alleati nel reclutamento e

RC: 93823

Disponível em: https://www.nucleodoconhecimento.com.br/economia- 
nella selezione del personale. Con mezzi tecnologici è possibile che le persone possano cercare lavoro ed essere selezionate.

L'utilizzo dei social network per la selezione del curriculum secondo Lago; Da Silva e Barbosa (2018) sta diventando sempre più reale, poiché, dall'utilizzo dei social network, c'è una maggiore portata delle persone per la selezione della posizione offerta, che consente di trovare il dipendente ideale per la posizione desiderata in azienda.

Queiróz e Sanches (2019, p. 472) menzionano che:

In concomitanza con le tecnologie, sono emersi i social network, dove è possibile prendere coscienza della vita personale delle persone in tempo reale; Twitter, Instagram, Facebook, difficilmente riesci a trovare qualcuno che non abbia almeno uno di questi social network in questi giorni. Per questo motivo, è comune che nei processi di selezione le reti siano utilizzate per disegnare un profilo personale del candidato in base ai loro posti, essendo un criterio per l'esclusione dei candidati.

Nella percezione di Melo (2014), i social network sono stati inizialmente visti come strumenti di incontri e riunioni in una società basata sulla distanza tra le persone dovuta alla globalizzazione. Tuttavia, i giorni nostri consentono ai social network di essere non solo per questi scopi, ma di estendere le loro concezioni ed evolversi consentendo la conoscenza e il contatto tra persone strane con specifici gruppi di interesse.

Un esempio dell'uso dei social network si osserva nel reclutamento online. II reclutamento online è uno strumento che differisce dal reclutamento tradizionale perché è una tecnica utilizzata, associata a tecnologie come Internet. Questo tipo di reclutamento mira a rivelare i posti vacanti e selezionare le persone solo attraverso reti virtuali (DE LIMA: RABELO, 2018).

Pedroso (2016, p. 25), descrive che:

RC: 93823

Disponível em: https://www.nucleodoconhecimento.com.br/economia- 
II reclutamento online è un metodo in rapida crescita in quanto sempre più persone rendono disponibile il loro Curriculum Vitae (CV) sul mercato online perché riconoscono il loro potenziale. II successo di questo metodo è intrinsecamente legato al fatto che le aziende possono raggiungere un pool più ampio di candidati con potenziale poiché le restrizioni geografiche sono meno significative, vale a dire che i singoli possono essere contattati da aziende da qualsiasi parte del mondo e possono richiedere un impiego da qualsiasi area del mondo.

Cassiano; Lima e Zupani (2016) identificano che il modo in cui avviene la diffusione sui social network per il verificarsi di assunzioni è considerato passivo. Questa forma di reclutamento avviene dalla diffusione del dipendente sui social media, uno di questi media si riferisce a Facebook e Instagram. Un altro reclutamento passivo coinvolge il social network di Linkedin, in questo social network il candidato viene selezionato dal suo riferimento di profilo in cui compaiono le sue qualifiche. Vale la pena notare che queste assunzioni sono fatte dai social network e che ogni social network porta una caratteristica differenziata sul candidato, quindi spetta al reclutatore sapere se sono diretti prima della scelta dei loro candidati alla selezione.

De Lima e Rabelo (2018, p. 06) affermano che:

\begin{abstract}
L'e-recruitment può anche fornire informazioni costanti ai candidati in merito alle opportunità di lavoro provenienti da diverse aree e organizzazioni. II reclutamento tradizionale è spesso pubblicato in annunci di giornali e riviste che richiedono un numero limitato di personaggi o informazioni. In questo senso, il reclutamento online può essere considerato una fonte di chiarezza e anche un modo dinamico, perché si possono diffondere link che indirizzano i candidati a contenuti pertinenti sull'organizzazione.
\end{abstract}

Lago; Da Silva Manhães e Barbosa (2018) menzionano nella loro ricerca che i social network e i media ottimizzano il tempo del reclutatore e la selezione delle persone. L'utilizzo di strumenti tecnologici migliora l'approssimazione tra l'azienda e il candidato portando agilità nella diffusione dei posti vacanti e nella ricezione dei curriculum da selezionare.

Secondo Oliveira (2013, p. 38):

RC: 93823

Disponível em: https://www.nucleodoconhecimento.com.br/economia- 
Buone relazioni interpersonali sono uno dei fattori che influenzano le prestazioni soddisfacenti delle produzioni quotidiane di un gruppo. Un team che lavora insieme per lo stesso obiettivo raggiunge con qualità ed efficienza ciò che viene proposto dal suo leader.

In questo senso, è essenziale che il manager conosca da vicino gli anidi e gli obiettivi di ogni dipendente per l'organizzazione. Questo in modo che non ci siano disagi legali quando si identifica qualcosa che non può portare benefici all'organizzazione, o anche al contrario, il dipendente è l'obiettivo di qualcosa di indesiderabile.

\section{CONSIDERAZIONI FINALI}

La ricerca svolta ha sollevato un argomento pertinente sull'assunzione e la selezione nelle organizzazioni nell'ambito dei cambiamenti derivanti dalla tecnologia. La gestione delle persone è un settore in cui l'intero processo delle risorse umane sviluppa, in questo ambiente, inizia la ricerca del dipendente ideale.

Visti i cambiamenti nella globalizzazione, il reclutamento e la selezione delle persone sono stati caratterizzati da nuove tecniche di scelta, tra queste nuove tecniche che fino ad allora erano faccia a faccia, i social network guadagnano spazio, abbattendo gli ostacoli geografici e espandendo la concorrenza per il posto vacante previsto.

Diventa evidente che l'uso delle tecnologie assume un ruolo rilevante nell'assunzione di questo dipendente, attraverso i social network, un potenziale strumento di reclutamento e selezione, rendendo le tecnologie un alleato nel processo decisionale.

Facebook, Instagram, Twitter, Linkedin e altri social network sono stati notati dai recruiter per trovare il candidato ideale. Tuttavia, ogni social network ha la sua caratteristica e che il reclutatore dovrebbe sapere come indirizzare ogni social network alla ricerca del candidato ideale per la propria azienda.

RC: 93823

Disponível em: https://www.nucleodoconhecimento.com.br/economia- 
Si conclude, quindi, che i social network sono strumenti tecnologici che aiutano la gestione delle persone alla ricerca dei loro candidati. Viene sottolineata l'importanza di estendere questo studio sulla base di prove scientifiche, in cui la ricerca può essere effettuata all'interno di aziende che utilizzano i social network per monitorare i propri dipendenti.

\section{RIFERIMENTI}

\section{ALBERTON, Luiz. Uma contribuição para a formação de auditores contábeis}

independentes na perspectiva comportamental. 2002. 270 f. Monografia (Programa de Pós-Graduação em Engenharia de Produção). Universidade Federal de Santa Catarina. 2002.

BARROS, Claudio Marcelo Edwards; DA SILVA, Pedro Ylunga Costa; VOESE, Simone Bernandes. Relação entre o custo da dívida de financiamentos e governança corporativa no Brasil. Journal of Accounting, Management and Governance, v. 18, n. 2, 2015.

CANALLI, Tiago; DE ANDRADE, Érica Fernanda Pereira. Recrutamento interno: uma nova perspectiva sobre plano de carreira. Revista Eletrônica Organizações e Sociedade, 2016, 5.3: 88-100.

CARVALHO, leda Maria Vecchioni. Recrutamento e seleção por competências. Rio de Janeiro: Editora FGV, 2015.

CASSIANO, Cecilia Nascimento; LIMA, Luciana Campos; DOS SANTOS ZUPPANI, Tatiani. A eficiência das redes sociais em processos de recrutamento organizacional. NAVUS-Revista de Gestão e Tecnologia, v. 6, n. 2, p. 52-67, 2016.

CHIAVENATO, Idalberto. Recursos humanos.7 ed. São Paulo: Atlas, 2002.

$\mathrm{RC}: 93823$

Disponível em: https://www.nucleodoconhecimento.com.br/economia- 
COSTA, Esdras da Silva et al. Análise das relações e ações conjuntas entre as empresas do APL têxtil da região metropolitana de São Paulo: contribuições para o seu crescimento. Interações (Campo Grande), v. 19, p. 401-415, 2018.

DE BEM NORO, Greice; STÜKER, Camila; DE OLIVEIRA, João Helvio Righi. A relação das características inerentes aos perfis de liderança no desenvolvimento do processo coaching. Revista Capital Científico-Eletrônica (RCCe) - ISSN 21774153, v. 13, n. 1, p. 59-76, 2015.

DE LIMA, Aryane Santos Henriques; RABELO, Aline Andrade. A importância do erecrutamento e seleção online no processo organizacional. Revista Psicologia,Diversidade e Saúde, v. 7, n. 1, p. 139-148, 2018.

DOS SANTOS, Márcia Gomes; DE OLIVEIRA, Rodrigo Cesar Reis; CENTURIÓN, Wanusa Campos. Recrutamento e seleção estratégicos: processos tradicionais e a influência das mídias sociais. Ideias e Inovação-Lato Sensu, v. 4, n. 3, p. 57, 2018.

KON, Anita. Ecossistemas de inovação: a natureza da inovação em serviços. Revista de Administração, Contabilidade e Economia da Fundace, v. 7, n. 1, 2016.

LAGO, Barbara; DA SILVA MANHÃES, Ana Claudia Tavares; BARBOSA, Marcus Vinicius. O uso de novas tecnologias digitais no recrutamento e seleção de talentos. Revista Interdisciplinar em Gestão, Tecnologia e Saúde, v. 1, n. 01, p. 161-178, 2018.

LEAL, Débora Ribas; MATTOS, Gisele Domingues de; FONTANA, Rosane Teresinha. Trabalhador com deficiência física: fragilidades e agravos autorreferidos. Revista brasileira de enfermagem, v. 66, n. 1, p. 59-66, 2013.

RC: 93823

Disponível em: https://www.nucleodoconhecimento.com.br/economia- 
LEITE, André Mourão. O impacto da transformação digital na gestão de pessoas: abordagens estratégicas para atrair, reter e motivar talentos da área de TI na região da grande Florianópolis. Gestão de pessoas-Unisul Virtual, 2019.

LIMA, Ricardo Leite; FIGUEIREDO, Giane Lourdes Alves de Souza. Novas estratégias do marketing 4.0 para as organizações. Facit Business and Technology Journal, v. 1, n. 21, 2020.

LORENZETTI, J. et al. Organização do trabalho da enfermagem hospitalar: abordagens na literatura. Texto Contexto Enferm., Florianópolis, v.23, n. 4, p. 110412, out-dez, 2014.

MELO, Cristiane Maria Freitas de. Freedom of expression on social networks: right to criticism of the employee versus image and honor of the employer. 2014. 136 f. Dissertação (Mestrado em Direito) - Pontifícia Universidade Católica de São Paulo, São Paulo, 2014.

MOTTA, Marlton Fontes, et al. O Uso das Redes Sociais no Ambiente de Trabalho e as Estratégias da Gestão de Pessoas para a Gestão de Resultados. In: Congresso de Gestão, Negócios e Tecnologia da Informação-CONGENTI. 2017.

MOZER, André Emílio. Recrutamento e seleção-uma proposta para recrutamento e seleção interno. Gestão em Foco: Revista Eletrônica On Line. Amparo: SP, 2016, 8.1 .

NOVATO, Douglas. 0 que é Governança de TI? OFICINA DA NET. 2014.Disponível em: https://www.oficinadanet.com.br/post/12712-o-que-egovernanca-de- Acesso em: 13/mai/2021

OLIVEIRA, Camila Miles; BUENO, Rose Ângela Vieira Passos. Uma análise da contribuição dos testes psicológicos no processo de recrutamento e seleção de

RC: 93823

Disponível em: https://www.nucleodoconhecimento.com.br/economia- 
pessoal. Simpósio de Produções Acadêmicas em Psicologia do Univag, n. 1, 2018.

OLIVEIRA, Rosane S. O papel do gestor na motivação dos seus colaboradores. Cairu em Revista. Jan 2013, Ano 02, nº2, p. 33-41, ISSN 22377719.

PEDROSO, Rita Cipriano. Redes sociais e recrutamento. 2016. 81 f. Tese de Doutorado. Universidade Católica de Portugal. Porto, Portugal, 2016.

PESSOA, Cláudio Roberto Magalhães et al. Da gestão de TI à gestão de informação e tecnologia: uma abordagem teórica da evolução do conceito= From management of technology and information to a management of information and technology: a theoretical approach of concept development. Descobrimentos da Ciência da Informação: desafios da Multi, Inter e Transdisciplinaridade (MIT): XVII Encontro Nacional de Pesquisa em Ciência da Informação (ENANCIB), 2016.

POCHMANN, Márcio. 0 emprego na globalização: a nova divisão internacional do trabalho e os caminhos que o Brasil escolheu. Bomtempo Editorial, 2015.

PORTO, Daniel da Silva et al. Gestão de pessoas: o caso da empresa Yes Contact Center no Município de Florianópolis/SC-Brasil. 2016. Dissertação de Mestrado.

QUEIRÓZ, Monique Mazon; SANCHES, Nathalia Paes. As influências das redes sociais no direito do trabalho. Revista do Tribunal Regional do Trabalho da 15a Região, n. 55, p. 271-280. 2019

REIS, Thompson Augusto, et al. Endomarketing, Liderança e Comunicação: reflexos na organização. Caderno Profissional de Marketing-UNIMEP, 2018, 6.1: 1-17.

RIBEIRO, Antonio de Lima. Gestão de pessoas. Saraiva Educação SA, 2017.

RC: 93823

Disponível em: https://www.nucleodoconhecimento.com.br/economia- 
SANTOS, Suênia Soares. Recrutamento e seleção: um estudo do impacto da tecnologia praticado por hotéis da orla de João Pessoa-PB. 2018. $44 \mathrm{f}$. Monografia (Graduação em Hotelaria). Universidade Federal da Paraíba. Paraíba. 2018.

SILVA, Bruno de Oliveira Fernandes da, et al. Teoria das Organizações. 2020. 46 f. USP - Universidade de São Paulo. Faculdade de Economia, Administração e Contabilidade - FEA-RP. São Paulo. 2020.

SILVA, Jessyca Rodrigues Henrique da. Impactos da tecnologia nos processos de gestão de pessoas: um estudo no setor hoteleiro. 2019. 129f. Dissertação (Mestrado em Turismo) - Centro de Ciências Sociais Aplicadas, Universidade Federal do Rio Grande do Norte, Natal, 2019.

SILVA, Vanessa; FERRETT, Kézia; MANCINI, Ronaldo. Recrutamento e Seleção: Um estudo sobre as técnicas utilizadas nas organizações. ETIC-ENCONTRO DE INICIAÇÃO CIENTÍFICA, 10.10. 2014.

SPADER, Gabriele A influência da competência profissional, da inteligência emocional, da produtividade e do engajamento sobre a satisfação com o trabalho em um ambiente de serviços de saúde. 2019. 93 f. Dissertação (Mestrado em Administração). Universidade de Caxias do Sul. 2019.

STECCA, Jaime Peixoto. Estratégias genéricas na gestão de pessoas e comprometimento organizacional: um estudo em sociedades cooperativas de crédito. 2014. 144 f. Tese. (Doutorado em Administração, Economia e Contabilidade). Universidade de São Paulo. 2014

TIDD, Joe; BESSANT, Joe. Gestão da inovação-5. Bookman Editora, 2015.

RC: 93823

Disponível em: https://www.nucleodoconhecimento.com.br/economia- 
WANZERLLER, Rafaela et al. Gestão de pessoas: visão estratégica sobre treinamento e desenvolvimento no contexto das organizações do século XXI. Revista de Administração e Negócios da Amazônia, v. 10, n. 2, p. 67-84, 2018.

Inviato: Maggio 2021.

Approvato: Luglio 2021.

RC: 93823

Disponível em: https://www.nucleodoconhecimento.com.br/economia- 